\title{
Maturational Hyperpigmentation: Cutaneous Marker of Metabolic Syndrome
}

\author{
Sidharth Sonthalia, ${ }^{1}$ Mahima Agrawal, ${ }^{2}$ Poonam Sharma, ${ }^{3}$ Amarendra Pandey ${ }^{4}$
}

1 SKINNOCENCE: The Skin Clinic \& Research Center, Gurugram, India
2 LHMC \& Associated Hospitals, New Delhi, India
3 Skin Institute \& School of Dermatology (SISD), New Delhi, India
4 Cosmasure Skin Hair, Fat Management and Laser Clinic, Jabalpur, India

Key words: maturational hyperpigmentation, facial melanosis, metabolic syndrome, facial acanthosis nigricans, dermoscopy

Citation: Sonthalia S, Agrawal M, Sharma P, Pandey A. Maturational hyperpigmentation: cutaneous marker of metabolic syndrome. Dermatol Pract Concept. 2020;10(2):e2020046. DOI: https://doi.org/10.5826/dpc.1002a46

Accepted: February 9, 2020; Published: April 20, 2020

Copyright: $@ 2020$ Sonthalia et al. This is an open-access article distributed under the terms of the Creative Commons Attribution License, which permits unrestricted use, distribution, and reproduction in any medium, provided the original author and source are credited.

Funding: None.

Competing interests: The authors have no conflicts of interest to disclose.

Authorship: All authors have contributed significantly to this publication.

Corresponding author: Sidharth Sonthalia, MD, DNNB, MNAMS, SKINNOCENCE: The Skin Clinic \& Research Center, C-2246, Sushant lok-1, Block-C, Gurugram-122009, India. Email: sidharth.sonthalia@gmail.com

\section{Case Presentation}

A 62-year-old Indian man with a strong family history of metabolic syndrome (MeTS) presented with asymptomatic, granular-surfaced, dark brown pigmentation on the cheeks (Figure 1, A and B). Nuchal/axillary acanthosis nigricans (AN) were conspicuously absent. Dermoscopy showed exaggerated light brown pseudoreticular network and scattered brown globules and structureless areas. At higher magnification $(\times 150)$, oval dark brown annular "ring"-like structures centered around hair follicles were observed (Figure 1C). The dermoscopic image was conspicuously devoid of sulci/cristae (which are considered typical of AN). Examination and biochemical evaluation revealed blood pressure $=160 / 102 \mathrm{~mm} \mathrm{Hg}$, waist circumference $=92 \mathrm{~cm}$, fasting blood glucose $=126 \mathrm{mg} / \mathrm{dL}$, and fasting serum triglycerides $=438 \mathrm{mg} / \mathrm{dL}$, confirming central obesity with MeTS. Histopathology revealed hypermelanized epidermis and dermis, and basal melanocytosis (Figure
1D). Clinicodermoscopicopathological correlation diagnosis was maturational hyperpigmentation $(\mathrm{MH})$ with MeTS.

\section{Teaching Point}

Although facial AN and MH may actually represent evolutionary standpoints on the morphological spectrum of cutaneous markers of MeTS (CMM) [1], labeling them as synonymous [2] without dermoscopy/supportive evidence seems inappropriate at present. Maturational hyperpigmentation is a newly described facial melanosis. It is a CMM akin to facial AN with which it does share some morphological features as well; however, specific features such as relatively softer surface with conspicuous but finer granularity and indistinct margins on gross morphology, and distinctive dermoscopy and histopathology [1], warrant identification of $\mathrm{MH}$ as a separate nosological entity. Thus, every physician's awareness of this relatively less evident and lesser-known asymptomatic entity is paramount to facilitate prompt workup for MeTS. 

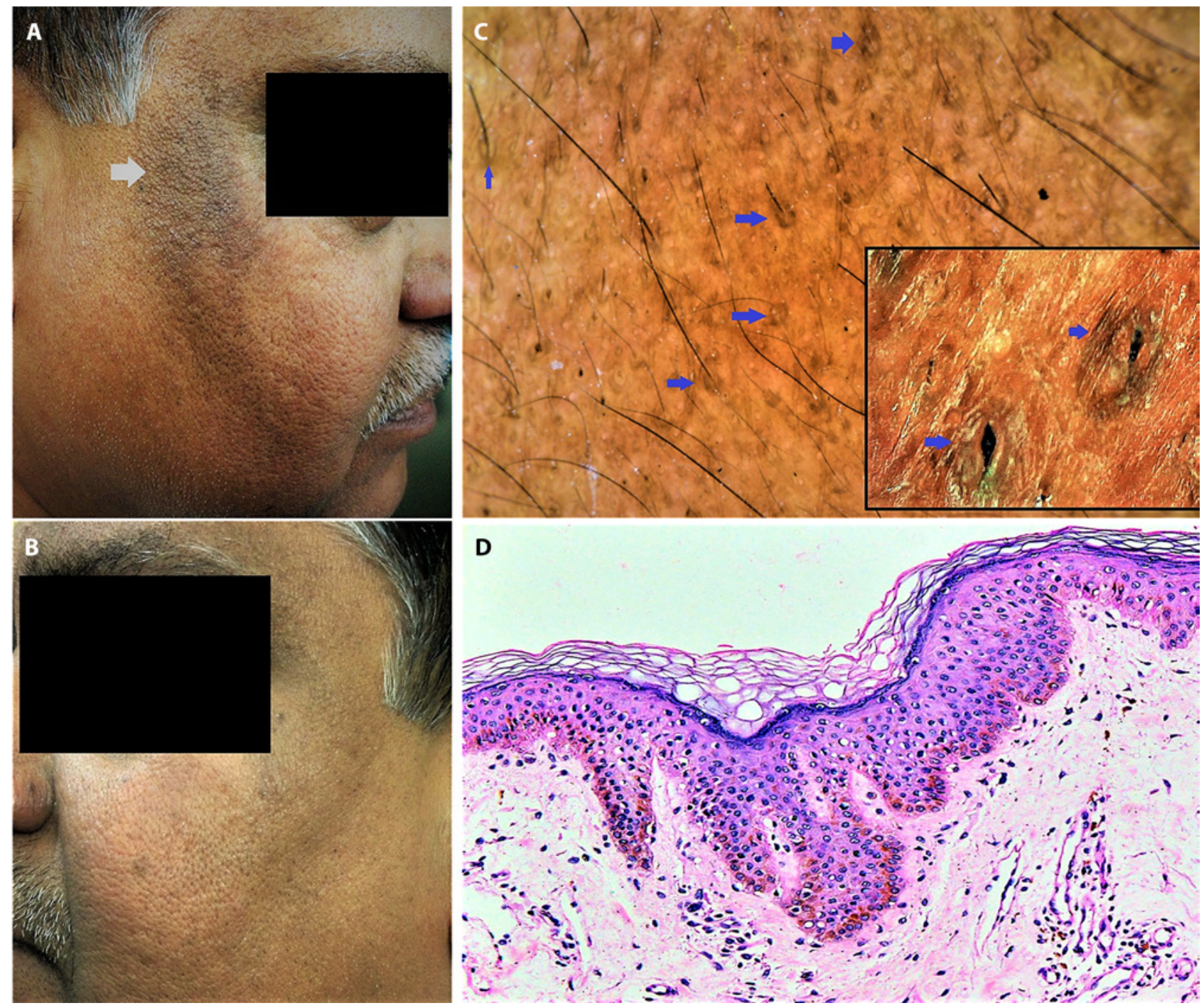

Figure 1. Maturational hyperpigmentation. (A) Patch of dark brown pigmentation with ill-defined margins and conspicuous granular surface over the right cheek (white arrow) extending from the angle of the eye to nearly the angle of the mouth. (B) Similar but much lighter pigmentation over the left cheek suggestive of an evolving lesion. (C) Videodermoscopic image of the hyperpigmentation on the right side revealing exaggerated light brown pseudoreticular network with scattered brown globules and structureless areas, perifollicular oval dark brown "rings" (blue arrows), and absence of sulci and cristae, the dermoscopic features that are pathognomonic of cutaneous acanthosis nigricans. The perifollicular rings can be appreciated better at higher magnification (blue arrows) in the figure inset (polarized, $\times 35$ and $\times 150$; Escope Videodermoscope, Timpac Healthcare Pvt. Ltd., New Delhi, India). (D) Histopathology showing basal cell layer melanocytosis, hypermelanization of basal and squamous layers of epidermis, and the dermis. Hyperkeratosis and papillomatosis are minimal-to-absent. H\&E, $\times 400$.

\section{References}

1. Sonthalia S, Sarkar R, Neema S. Maturational hyperpigmentation: clinico-dermoscopic and histopathological profile of a new cutaneous marker of metabolic syndrome. Pigment Int. 2018;5(1):54-56.

2. Verma S, Vasani R, Joshi R, Phiske M, Punjabi P, Toprani T. A descriptive study of facial acanthosis nigricans and its association with body mass index, waist circumference and insulin resistance using HOMA2 IR. Indian Dermatol Online J. 2016;7(6):498-503. 